\title{
XVII.
}

Aus dem pharmakologischen Institut zu Bonn.

\section{Die Einwirkung des Alkohols auf den Gaswechsel des Menschen.}

\author{
Von \\ Dr. J. Geppert, \\ Assistent am pharmakologischen Institut.
}

Die Wirkung des Weingeistes auf den animalen Gaswechsel ist seit langer Zeit und von den verschiedensten Seiten studirt, aber; soweit wenigstens exacte ${ }^{1}$ ) Arbeiten in Frage kommen, sind diese Untersuchungen bisher nur an Thieren ${ }^{2}$ ) angestellt worden, und selbst wenn die verschiedenen Experimentatoren zu iibereinstimmenden Resultaten gekommen wären, was nicht der Fall ist, so würde es nicht gestattet sein, die bislang gewonnenen Resultate ohne Weiteres auf den Menschen zu übertragen. Denn zunächst wissen wir durch lange Reihen von Erfahrungen klinischer und experimenteller Natur, dass dasselbe Gift sehr verschieden auf die verschiedenen Thierklassen wirkt; und dann kam im vorliegenden Falle speciell nocb ein zweiter Factor in Betracht: die Gewöhnung des Menschen an

1) Prout (Lancet 1843. Vol. II. 17), sowie Vierordt (Physiologie des Athmens mit besonderer Rücksicht auf die Kohlensäure.), Eduard S m it h (Lancet 1861. Januar) und noch Andere fanden alle beim Menschen eine mehr oder minder grosse Herabsetzung der Kohlensäureausscheidung nach Alkoholgenuss. Indess ist die Methodik ihrer Arbeiten wenig zuverlässig.

2) v. Boeck und Bauer (Ueber den Einfluss einiger Arzneimittel auf den Gasaustausch bei Thieren. Zeitschrift für Biologie. 1874. X. Bd. S. 361) fanden bei Hunden nach kleinen Dosen Alkohol stets eine Verminderung der Kohlensäureausscheidung, dagegen eine Vermehrung nach grossen Dosen. $\mathrm{Zuntz}$ und Wolfers (Archiv für die gesammte Physiologie. 1883. XXXII. Bd. S. 255-279) fanden am Kaninchen fast stets ein Steigen des Gaswechsels, selten Gleichbleiben.

In veuester Zcit fanden dann Bodländer und Fath an Hunden und Kaninchen, dass Alkoholgenuss den Gaswechsel entweder unberührt lasse, oder herabsetze (Ueber den Einfluss des Weingeistes auf den Gaswechsel, von Dr. G. Bodlănd er. Zeitschr. f. klin. Med. XI. Bd. Hett 5-6. S. 548. 1886). Pharmakolog. Institut zu Bonn. 
den Alkoholgenuss, ein Factor, dessen Wirksamkeit überhaupt nicht zu berechnen war. Unter diesen Umständen folgte ich gern der Anregung des Herrn Geh. Rath $\mathrm{B}$ in $\mathrm{z}$, die Frage nach dem Einfluss des Alkohols auf den Gaswechsel des Menschen einer experimentellen Prüfung zu unterziehen.

\section{Die Methodik.}

Die Methoden, welche es bisher gab, den Gaswechsel des Menschen zu studiren, reichten nicht aus, um die vorliegende Frage zu lösen. Denn die klassische Pettenk of er'sche Methode, der wir sonst fast alle unsere Kenntnisse iuber den menschlichen Gaswechsel verdanken, gestattet eine scharfe Bestimmung nur für die Kohlensäure, im vorliegenden Falle war eine Bestimmung auch des Sauerstoffconsums in hohem Grade wiinschenswerth.

So wählte ich denn eine andere Methode, die ursprtinglich von Zuntz und mir für Thierversuche erdacht, sich leicht auf den Menschen übertragen lässt. Das Princip derselben ist folgendes:

Kennt man die Menge und chemische Zusammensetzung des von einem Thier in- und exspirirten Gasgemisches, so kann man aus diesen Factoren den respiratorischen Gaswechsel bestimmen. Aus der Zusammensetzung der Exspirationsluft gegenüber der Inspirationsluft berechnet man, wieviel Procent Sauerstoff in den Lungen zurïckgehalten, wieviel Procent Kohlensäure abgegeben sind, und diese Zahlen multiplicirt mit der durch die Lungen passirten Gasmenge ergeben Sauerstoffverbrauch und Kohlensäurebildung. Nun schwankt die Zusammensetzung der (Inspirations-) Luft nach den besten vorliegenden Versuchen ${ }^{1}$ ) in so geringen Grenzen, dass sie für den vorliegenden $\mathrm{Zweck}$ als constant angesehen werden kann. Es bleiben demnach nur zwei Factoren zu bestimmen: die Menge der exspirirten Luft und ihre Zusammensetzung.

Die Menge wird an einer Gasuhr gemessen, durch die die ausgeathmete Luft hindurch streicht, die chemische Zusammensetzung an Proben bestimmt, die vom Exspirationsstrom abgesaugt werden. Die Absaugung ist in der Weise regulirt, dass die Menge der zur Untersuchung abgenommenen Gasproben in bestimmtem procentischen Verhältniss zur Gesammtmenge steht.

Wie aus dem Gesagten ersichtlich, ist es nöthig, die gesammte exspirirte Luft durch die Gasuhr zu leiten. Beim Thier erreicht man diesen Zweck in bekannter Weise, indem man durch Trachealcantile und Ventile athmen lässt, beim Menschen ersetzt die Stelle.

1) Kreussler, Landwirthschaftliche Jahrbücher. 14. Bd. S. 307. 
der Trachealcanüle ein Mundstück, welches nach Verschluss der Nase Iuftdicht zwischen Lippen und Kiefer eingelegt wird.

Die Ausführung der eben im Princip angegebenen Methode ist folgende:

Die Ventile bestehen aus etwa 1//2 Liter grossen gläsernen Pulverflaschen, in welche luftdicht breite, starke Gummistopfen eingefügt sind. Jeder Gummistopfen trägt zwei $2 \mathrm{~cm}$ breite Durchbohrungen, durch welche passende Glasröhren hindurchgehen. An je einer dieser Glasröhren ist ein Stück Rinderdarm, das in Glycerin gelegen hatte, befestigt. Jedes der so ausgeristeten Pulvergläser stellt demnach ein Ventil dar, welches durch das mit Darm beschickte Glasrohr nur die Exspiration, durch das andere nur die Inspiration gestattet. Diese Ventile spielen ausserordentlich leicht und bewirken in der That bei der Athmung kein bemerkbares Hinderniss. Das Mundstiick, welches ich verwandte, ist von ähnlicher Construction, wie es bei den pneumatischen Apparaten verwandt wird; es besteht zunächst aus einer Hartgummiplatte, welche der Krïmmung des Oberkiefers conform gebildet ist; in diese ist eine runde $2 \mathrm{~cm}$ breite Oeffnung geschnitten, in welche ein T-Sttick mit $2 \mathrm{~cm}$ breiten Hartgummiröbren passt. Die beiden freien Enden des T-Stiekes sind durch Gummischläuche mit den Ventilen verbunden. In den Gummischläuchen liegen Drahtspiralen, um das Zusammenknicken derselben zu hindern. Wird nun die Hartgummiplatte zwischen Lippen resp. Backen und die Kiefer geschoben, so bilden die anliegenden Weichtheile einen luftdichten Abschluss und In- und Exspiration gehen nach Verschluss der Nase durch die Ventile. Die Schläuche werden durch Klammern in passender Lage fixirt, so dass das Mundstiick ohne Zerrung im Munde hängt. Der Verschluss der Nase wird am besten in der Weise ausgeführt, dass man Baumwollebäusche, in Vaselin getränkt, mit der Pincette fest in dieselbe einführt.

Vom Exspirationsventil geht ein Schlauch zur Gasuhr.

Jede Umdrehung der angewandten Gasuhr (ein Experimentirgasmesser von S. E1ster, Berlin) entsprach einem Durchgang von 40 Liter. Die Aichung ergab, dass die Fehler der Gasuhr so gering waren (ca. $1 / 2$ Proc.), dass sie für den vorliegenden $Z_{w e c k}$ nicht in Frage kommen.

Es ist endlich noch nöthig, die Methode zu beschreiben, welche ich $^{1}$ ) anwandte, um Gasproben proportional der durchgehenden Luftmenge vom Exspirationsstrom abzusaugen. Die Gasuhr wird in der

1) Zuntz und ich hatten ursprünglich eine andere Methode zu gleichem Zwecke verwandt. 
von Voit für Ventilationszwecke angegebenen Weise construirt, d. h. das freie hintere Ende der Gasuhraxe ist vierkantig ausgezogen. Auf diesen Vierkant wird ein leichtes Rad mit breitem Rand aufgesetzt. Um das Rad ist eine Schnur gelegt, so dass bei jeder Drehung sich ein bestimmter Theil derselben abwickelt. Hinter der Gasuhr und unterhalb des Niveaus ihrer Axe ist senkrecht eine Glasröhre eingespannt. Diese läuft oben und unten in je zwei andere Röhren aus. Die oberen sind capillarer Natur (2 $\mathrm{mm}$ licht), die unteren etwas weiter. An beide untere Fortsätze werden lange Schläuche befestigt, der eine trägt am freien Ende eine Füllkugel, der andere eine dünne kleine Glasröhre mit Hahn. An diese Glasröhre wird mit mehreren Drahtringen der freie Theil der vom Rade kommenden Schnur befestigt. Durch die Füllkugel wird zunächst der ganze Apparat mit Quecksilber gefulltt und dann der zur Füllkugel gehende Schlauch durch eine Klemme abgesperrt. Ist der zur kleinen Glasröhre gehörige Hahn offen, so stellt sich jetzt das Quecksilber in der grossen Glasröhre auf gleiches Niveau ein mit der oberen Oeffnung der kleinen Glasröhre; sinkt bei der Drehung des Rades mit der Abwicklung der Schnur die kleine Glasröhre, so fliesst das Quecksilber aus der grossen Glasröhre durch den Schlauch und die kleine Glasröhre und tropft aus ihrer oberen freien Oeffnung heraus. Da die Abwicklung der Schnur proportional der Raddrehung und diese wieder gleich der Axendrehung der Gasuhr und damit auch proportional der durchgehenden Luftmenge ist, so fliesst demnach das Quecksilber aus der grossen Glasröhre in einer der Bewegung der Gasuhr proportionalen Menge aus, d. h. werden grosse Luftquantitäten geathmet, so werden relativ grosse Quecksilbermengen ausfliessen, werden kleine Luftquantitäten geathmet, so fliessen geringe Quecksilbermengen aus. Die directe Messung des ausgeflossenen Quecksilbers, verglichen mit dem Gang der Gasuhr, bestätigte dann die Richtigkeit der Berechnung. Die capillaren Fortsätze am oberen Ende der grossen Glasröhre dienen zu folgendem Zweck: Der eine fuhhrt vermittelst eines passenden capillaren Verbindungsstiickes in das Exspirationsventil, der andere in eine Quecksilberwanne. Beide sind mit Hähnen versehen. Ist nun der zum Exspirationsventil gehende Hahn geöffnet, der andere geschlossen, so wird, wenn das Quecksilber bei der Raddrehung aus der grossen Glasröhre ausfliesst, aus dem Exspirationsventil die Luft angesaugt. Wie aus der Construction ersichtlich, würde der vom Rad niederbängende, mit Quecksilber gefulltte Schlauch einen Zug an der Axe der Gasuhr ausüben. Um zunächst die Hauptlast des Schlauches za übernehmen, ist in gleicher Höhe mit der Mitte der 
grossen Glasröhre eine Klammer angebracht, die den Schlauch fest umspannt. Der jetzt noch iibrig bleibende, überhaupt nicht mehr sehr schwerwiegende Theil des Schlauches wird contrebalancirt durch ein leichtes Bleigewicht, welches an einem Faden vom Rade niederhängt. Der Faden ist im umgekehrten Sinne um das Rad geschlungen, als der oben beschriebene. ${ }^{1}$ ) Wie aus der Beschreibung ersichtlich, hat man es nun in der Hand, die Quecksilbermasse, welche in der Zeiteinheit ausfliessen soll, beliebig gross oder klein zu machen, indem man entweder das Rad an der Gasuhr vergrössert, oder aber den Durchmesser der grossen Glasröhre verändert.

Für den Menschen wandte ich stets ein $\operatorname{Rad}$ von $5 \mathrm{~cm}$ Durchmesser und eine Glasröhre von $1 \mathrm{~cm}$ Durchmesser und $40 \mathrm{~cm}$ Länge an. Diese füllt sich in etwa 15-20 Minuten.

Die abgesangte Gasprobe wird für die Analyse in ein Eudiometer übergeführt. $\mathrm{Zu}$ diesem Zweck schliesst man zunächst sänımtliche Hähne des Apparates ab, so dass weder Quecksilber aus, noch Luft in die grosse Glasröhre eintreten kann. Alsdann öffnet man die Klammer des zur Fullkugel gehörigen Schlauches und setzt das Gas im Rohr unter Ueberdruck. Ueber die Mündung der Capillare in der Quecksilberwanne wird ein Eudiometer gesetzt und nun nach Oeffnung des zu dieser Capillare gehörigen Hahnes das Gas in das Eudiometer übergetrieben. Die Analyse wird nach den von mir gegebenen Methoden ${ }^{2}$ ) ausgefubhrt, die Kohlensäure mit 3-4 Proc. Kalilauge absorbirt, der Sauerstoff durch Verpuffung mit Wasserstoff bestimmt.

Im Eudiometer befand sich bei Einlass des Gases bereits eine mindestens $1 \mathrm{~cm}$ hohe Wasserschicht. Sauerstoff und Stickstoff und die höchstens 4 proc. Kohlensäure, aus welchen die Exspirationsluft besteht, haben einen so geringen Absorptionscoefficienten in Wasser, dass diese Anordnung keinen nachweislichen Fehler bedingen konnte. Die Vortheile derselben sind 1. c. von mir besprochen. Im vorliegenden Falle hatte sie noch einen anderen Zweck. Falls Alkoholdampf in die Exspirationsluft uberging, was ja nur in sehr geringer Menge geschieht ${ }^{3}$ ), löste er sich in diesem Wasser auf und

1) Ideell wechselt ja die Schwere des noch restirenden Schlauchendes mit den verschiedenen Positionen desselben. Indess sind diese Unterschiede ausserordentlich gering.

2) Geppert, Die Gasanalyse und ihre physiologische Anwendung. Berlin 1885. Verlag von Hirschwald.

3) Siehe C. Binz, Die Ausscheidung des Weingeistes durch Nieren und Lungen. Dieses Archiv. 1877. VI. Bd. S. 287. Fortsetzung dieser Untersuchungen vgl. Bodländer, Archiv für die gesammte Physiologie. 1883. 32. Bd. S. 398. 
schädigte so nicht weiter die Analyse. Es lässt sich nun nachweisen, dass factisch im Eudiometer kein Alkoholdampf mehr enthalten war. Nach mehreren der stärksten Alkoholdosen, die ulberhaupt verabreicht wurden, wurde die abgenommene Gasprobe in der Weise untersucht, dass unmittelbar nach der Verpuffung (d. h. also etwa 3 bis 4 Minuten nach derselben, denn so viel Zeit ist nöthig, um den grossen Glascylinder mit Wasser zu füllen, das Eudiometer zu richten u. s. w.) die Gasmenge unter allen denkbaren Cautelen scharf bestimmt wurde; dann wurde nach 24 stlindigem Warten eine zweite Bestimmung gemacht. Wäre nun Alkoholdampf im Eudiometer gewesen, so wäre bei der Verpuffung Kohlensäure entstanden, die von der im Eudiometer befindlichen schwachen Kalilauge langsam absorbirt worden wäre; das Volumen wäre also bei der zweiten Ablösung etwas kleiner ausgefallen. Dies war indess nie der Fall.

Die Berechnung des verbrauchten Sauerstoffes und der ausgegebenen Kohlensäure wird in folgender Weise angestellt. Zunächst wird die durch die Gasuhr gegangene feucht gemessene Luftmenge auf $0^{0}, 76 \mathrm{~cm}$ Quecksilberdruck und Trockenheit nach bekannter Formel reducirt. Die gebildete Kohlensäure ergiebt sich aus der so berechneten Zahl und aus der durch die Analyse gefundenen $\mathrm{Zu}$ sammensetzung der Exspirationsluft durch einfache Multiplication. Etwas umständlicher ist die Berechnung des verbrauchten Sauerstoffes. Das verbrauchte Sauerstoffquantum ist gleich der in die Lungen eingefuhrten minus der ausgefthrten Sauerstoffmenge. Die ausgeführte Sauerstoffmenge ist leicht berechnet, sie ist gleich der ausgeathmeten Gasmasse, multiplicirt mit dem procentischen Antheil, den der Sauerstoff an derselben hat. Umständlicher ist die Berechnung der eingefthrten Sauerstoffmenge. Die Inspirationsluft besteht aus 79,07 Theilen Stickstoff und 20,93 Theilen Sanerstoff. Exspirirt wird genau das gleiche Quantum Stickstoff, wie inspirirt, und zwar ist das exhalirte Stickstoffquantum gleich der Gesammtmenge des ausgeathmeten Gases multiplicirt mit dem analytisch gefundenen Procentgehalt desselben an Stickstoff. $\mathrm{Zu}$ dieser so berechneten, eingeführten Stickstoffmenge steht nach dem Gesagten die eingeführte Sauerstoffmenge im Verhältniss $\frac{79,07}{20,93}$ und es ist daher durch Multiplication dieses Bruches mit der gefundenen Stickstoffmenge die eingefibrte Sauerstoffmasse bestimmt.

In praxi empfiehlt es sich, die angegebene Rechnung etwas zu modificiren. Man berechnet zunächst, wie viel Sauerstoff im Exspirationsgas enthalten sein würde, wenn überhaupt in den Lungen kein 
solcher aufgenommen wäre. Dies ist, wenn die gefundene procentische Stickstoffmenge $=\mathrm{N}$ ist $=\mathrm{N} \frac{79,07}{20,93}$. Von dieser Zahl wird abgezogen die factisch gefundene procentische Sauerstoffmenge des Exspirationsgemisches, diese Differenz ist das Sauerstoffdeficit. Dies multiplicirt mit der gesammten exspirirten Gasmasse ergiebt den Sauerstoffverbrauch.

Berechnet man nun, wie hoch ungefähr die Fehlergrenze der Methode gehen wird, so ist es evident, dass dies zunächst davon abhängt, wie scharf Sauerstoff, Kohlensäure und Kenge der Exspirationsluft bestimmt sind. Die Fehler der Gasanayse überschreiten 0,05 Proc. nicht, der Fehler der Gasuhr beträgt etwa 0,5 Proc. Bedenkt man noch, dass die geringe Kohlensäuremenge der atmosphärischen Luft (0,03 Proc.) und die kleinen Schwankungen im Sauerstoffgehalt derselben nicht berücksichtigt sind, so ergiebt sich, dass der Fehler der Bestimmung für Sauerstoff und Kohlensäure im Ganzen ungefähr 0,1 Proc. betragen wird. Im Durchschnitt enthält nun die Exspirationsluft etwa 3,5 Proc. Kohlensäure und das Sauerstoffdeficit beträgt etwa 4,2 Proc. Demnach ist der Fehler $=1 / 35$ resp. $1 / 42$, d. h. also etwa $21 / 2-31 / 2$ Proc., einschliesslich des Fehlers der Gasubr.

Um nun festzustellen, ob die geschilderte Methode wirklich die berechnete Schärfe besitzt, machte ich einen Controlversuch nach folgendem Princip:

Die Kohlensäuremenge, welche ein tracheotomirtes, durch Ventile athmendes Kaninchen ausscheidet, wurde in Kalilauge aufgefangen. Vor den Kaliröhren war der oben geschilderte Respirationsapparat eingeschaltet und nach der angegebenen Methode wurde e benfalls die ausgeathmete Kohlensäure bestimmt. Das Exspirationsventil fasst entsprechend der geringen Grösse der Kaninchenathmung etwa $20 \mathrm{ccm}$. Nur in zwei unwesentlichen Punkten musste von der oben geschilderten Anordnung abgewichen werden: da die Exspiration unter Druck stattfindet, um die Luft durch die mit Kalilauge gefüllten Röhren zu treiben, zog ich vor, für die Inspiration ein Spritzflaschenventil zu verwenden; und die Gasubr wurde vor dieses gelegt. Da die exspirirte Gasmenge fast genau gleich der inspirirten ist, so kommt der durch diese Anordnung bewirkte Fehler der Messung der Gasmenge in der That nicht in Betracht. Die grosse Gasuhr, an welcher der geschilderte Absaugeapparat befestigt ist, ist fur den vorliegenden Versuch, bei welchem es sich doch nur um relativ kleine Gasmengen handelt, nicht hinreichend genau, deshalb wurde vor dieselbe noch ein kleiner Experimentirgasmesser zur Controle eingeschaltet. 
Die Kalilauge (specifisches Gewicht $=1,25$ ) befand sich in zwei 1,10 m langen, $16 \mathrm{~mm}$ breiten Glasröhren. Hinter den Kaliröhren befand sich noch ein mit Barytwasser gefülltes Spritzflaschenventil. $\mathrm{Da}$ das Kaninchen nicht vermochte, die exspirirte Luft durch die Kaliröhren zu treiben, wurde am Ende der Leitung vermittelst einer Wasserstrahlpumpe ein constanter schwacher, negativer Druck hervorgebracht, so schwach jedoch, dass die Exspiration immer noch unter starkem positiven Druck von Statten ging. Die zweite Kaliröhre nahm be dem Versuch nur noch ein sehr geringes Quantum Kohlensäure au i, Jas Barytwasser blieb vollkommen klar. Die Kalimenge in beiden Röhren war genau bestimmt. Ihr Kohlensäuregehalt vor und nach dem Versuch wurde durch Entgasung von Proben in der Quecksilberpumpe in bekannter Weise bestimmt. Der Versuch gab nun folgende Daten:

Die Kohlensäureausscheidung des Kaninchens wird gleichzeitig nach beiden Methoden gemessen in der Zeit von $4 \mathrm{~h} .28 \mathrm{~m}$. bis $5 \mathrm{~h}$. $19 \mathrm{~m}$. Das Thier hatte $0,5 \mathrm{~g}$ Chloralhydrat erhalten. Die Athemgrösse war pro Minute:

$$
\begin{aligned}
& \text { Von } 4 \mathrm{~h} 28 \mathrm{~m} \text { bis } 4 \mathrm{~h} 33 \mathrm{~m}=314 \mathrm{ccm} \\
& =4 \mathrm{~h} 33 \mathrm{~m}=4 \mathrm{~h} 36 \mathrm{~m}=330= \\
& =4 \mathrm{~h} 36 \mathrm{~m}=4 \mathrm{~h} 40 \mathrm{~m}=385= \\
& =4 \mathrm{~h} 40 \mathrm{~m}=4 \mathrm{~h} 44 \mathrm{~m}=232= \\
& =4 \mathrm{~h} 44 \mathrm{~m}=4 \mathrm{~h} 50 \mathrm{~m}=306= \\
& =4 \mathrm{~h} 50 \mathrm{~m}=5 \mathrm{~h} 4 \mathrm{~m}=285= \\
& =5 \mathrm{~h} 4 \mathrm{~m}=5 \mathrm{~h} 16 \mathrm{~m}=311= \\
& =5 \mathrm{~h} 16 \mathrm{~m}=5 \mathrm{~h} 19 \mathrm{~m}=283=
\end{aligned}
$$

Im Ganzen wurden von 4 h. 28 m. bis 5 h. 19 m. 15,520 Liter Luft bei einem Barometerstande von $756 \mathrm{~mm}$ und 20,40 Temperatur geathmet. Die abgesaugte Luftprobe betrug $105,3 \mathrm{ccm}$. Sie enthielt 3,73 Proc. Kohlensäure. Berechnet man nach diesen Daten die ausgeschiedene Kohlensäure, so erhält man 520,6 ccm.

In beiden Röhren befanden sich $250 \mathrm{ccm}$ Kalilauge. Diese enthielten ursprünglich $461,8 \mathrm{ccm}$ Kohlensäure. Nach Schluss des Versuches enthielten sie $986,5 \mathrm{ccm}$; vom Thiere waren abgegeben 986,5 $-461,8=524,7 \mathrm{ccm}$ Kohlensäure. Es wurden jedesmal zur Probe $50 \mathrm{ccm}$ Kalilauge entgast. Die Uebereinstimmung beider Methoden (520,6:524,7 $\mathrm{cm}$ ausgeschiedener Kohlensäure) ist so gross, als sie irgend erwartet werden konnte.

Es ist noch nöthig, Einiges über die Vorsichtsmaassregeln anzuführen, die man bei Anwendung der Methode am Menschen zu beobachten hat. Die Versuchsperson muss ganz bequem auf einem 
Sopha liegen oder sitzen; sobald die Lage eine gezwungene oder unangenehme ist, resultiren unwillkürliche Muskelbewegungen, welche den Sauerstoffverbrauch erheblich steigern können. Nach Beginn der Athmung am Apparat folgen rneist einige Minuten, in denen die Athmung noch unregelmässig ist, d. h. wenn man die binnen $1 \mathrm{Mi}$ nute geathmete Luftmenge an der Gasuhr abliest, so zeigt sich, dass die Lungenventilation nicht eine constante ist, meist sinkt sie langsam ab. Indess nach kurzer Zeit verschwindet diese Unregelmässigkeit. Die ruhige normale Athmung, welche sich jetzt einstellt, schwankt innerhalb relativ kleiner Grenzwerthe auf und nieder; betrachtet man genau, so erkennt man deutlich die Gesetzmässigkeit dieser Schwankungen.

Die Athmung am Apparat ging stets leicht und mühelos, wurde stundenlang ohne Beschwerden ertragen. Von Zeit zu Zeit liest man die Werthe an der Gasuhr ab. Es hat dies wesentlich den Zweck, einen objectiven Anhaltspunkt dafür zu haben, dass die Versuchsperson in der That ruhig gelegen und keine wesentlichen Muskelactionen gemacht hat. Jede Muskelaction treibt die Athmung deutlich in die Höhe und ein solcher abnormer Werth tritt dann sehr scharf aus der Reihe der ïbrigen normalen Werthe hervor.

Die Vorrichtung zum Absaugen der Luftprobe wird so eingestellt, dass nach der Füllung mit Quecksilber und Schluss sämmtlicher Hähne die Schnur so weit um das Rad gelegt ist, dass die kleine mit einem Hahn versehene Glasröhre über dem Niveau des höchsten Punktes der grossen Glasröhre steht. Bei der Athmung sinkt sie langsam herab. Steht die obere Oeffnung der kleinen Glasröhre ungefähr im Niveau des oberen Endes der Quecksilbersäule im grossen Glasrohr, so öffnet man den zum Exspirationsventil führenden Hahn und den Hahn der kleinen Glasröbre, liest im Moment, wo die ersten Quecksilbertropfen fallen, die Gasubr ab und notirt die Zeit genau.

Der Plan, nach welchem die Alkoholversuche unternommen wurden, war folgender: Zunächst wurde der normale Gaswechsel der Versuchsperson in der Ruhe bestimmt. Stets wurde gewartet, bis seit der letzten genommenen Mahlzeit einige Stunden verflossen waren. Oefters wurde eine zweimalige Normalbestimmung am selben Tage, eine dicht vor der Mittagsmahlzeit, die andere einige Stunden nach derselben, vorgenommen.

War der normale Gaswechsel bestimmt, so wurde die betreffende Dosis Alkohol möglichst schnell getrunken und dann sofort wiederum 
der Gaswechsel bestimmt. Die Zeit, welche der ganze Versuch in Anspruch nimmt, variirt etwas. Liegt die Versuchsperson sehr ruhig, so kann man sich eventuell mit ein er Normalbestimmung begnügen, welche sich etwa über $30-40$ Minuten ausdehnt, anderenfalls thut man gut, eine Controlbestimmung hinzuzuftugen. Wie lange nach dem Einnehmen des Alkohols die Bestimmungen fortzufuhren sind, war nicht a priori zu entscheiden; ursprünglich beobachtete ich noch stundenlang nachher, bei späteren Versuchen kürzte ich aus Grunden, die aus den Versuchen selbst sich ergeben haben, diese Zeit wesentlich $a b$; das Genauere über jeden einzelnen Versuch befindet sich in den im Anhang gegebenen Daten.

Der Alkohol wurde bei den ersten Versuchen als Alkohol absolutus verdünnt mit der hinreichenden Quantität Wasser und etwas Zucker gegeben, bei den späteren Experimenten wurde er auch in Gestalt von Wein und Cognac gereicht.

Es zeigte sich sehr bald, wie dies auch a priori zu erwarten war, dass die vorliegende Untersuchung mit Zuverlässigkeit nur innerhalb gewisser Grenzen ausführbar ist. Denn, sobald man stärker berauschende Dosen von Alkohol giebt, so kann man zwar wohl noch den Gaswechsel hinreichend scharf bestimmen, aber die Experimente werden fast stets werthlos, denn entweder werden die Versuchspersonen unruhig, werfen sich hin und her oder sie verfallen in Schlaf. Je nachdem das Eine oder das Andere stattfindet, erhält man dann ein Plus oder Minus, aber dies sind dann erst secundäre Folgen der Alkoholwirkung und haben mit dem reinen Effect derselben auf den Körper nichts gemein. Um daher uberhaupt die Effecte sehr grosser Alkoholdosen studiren zu können, blieb nichts übrig, als einem Gewohnheitstrinker eine grosse Dosis Alkohol zu geben, wie er sie noch gerade vertrug. Eine solche Reihe ist in den mitgetheilten Versuchen enthalten.

Im Ganzen habe ich an 4 Personen experimentirt. Zwei derselben (Kr. und Me.) waren an mässigen, einer (Mo.) überhaupt nicht an Alkoholgenuss gewöhnt. Li. hingegen war Gewohnheitstrinker.

Gehen wir nunmehr zu den gewonnenen Resultaten über, so ist es zunächst interessant, einen Blick auf die Aufschlüsse zu werfen, die die angewandte Methode für die normale menschliche Respiration geliefert hat.

Die aus sämmtlichen Ruhewerthen gezogenen Mittel ergaben, dass Me., welcher von allen Versuchspersonen der fettreichste war, den geringsten Sauerstoffverbrauch (auf 1 Kilo Gewicht und 1 Minute bezogen) hatte, nämlich $3,27 \mathrm{ccm}\left(\mathrm{CO}_{2}=2,28\right)$. 
Li., der der Musculöseste und an die schwerste Arbeit Gewöhnte war, hat pro Kilo Gewicht und Minute einen Sauerstoffverbrauch von 4,41 ccm. Kr., ein graciler Mensch, dessen Körperbau nichts besonders Bemerkenswerthes darbot, hatte pro Kilo und Minute einen Sauerstoffverbrauch von $3,53 \mathrm{ccm}\left(\mathrm{CO}_{2}=2,88 \mathrm{ccm}\right)$.

Die Kohlensäurewerthe entsprechen den niedrigsten, welche nach der Pettenk of er'schen Methode gefunden sind, resp. sind noch etwas geringer als diese. Der Grund ist wohl, dass bei den mitgetheilten Versuchen in der That die Versuchspersonen so ruhig lagen, wie es im wachen Zustand möglich ist. Als mittlerer Sauerstoffconsum des Menschen in der Ruhe würde sich also ungefähr pro Kilo und Minute eine Grösse von 3,5-4,0 cem ergeben.

Die Ruhewerthe, welche an verschiedenen Tagen gewonnen wurden, weichen nicht unwesentlich von einander ab; bei $\mathrm{Kr}$. und Me. um ungefähr 15-20 Proc. des Gesammtgaswechsels, bei Li. um ungefähr 10 Proc. Es kann dies Schwanken nicht überraschen, wenn man bedenkt, wie die verschiedenen Erregungszustände der glatten Musculatur, der Drïsen $u$. s. w. auf den Sauerstoffconsum wirken, wie ferner der wechselnde, aber doch stets vorhandene Tonus der Körpermusculatur die Oxydation beeinflussen muss, Functionen, die von der Reizung peripherer Nerven, der Erregbarkeit des Centralnervensystems u. s. w. abhängen und gänzlich unserer Willkür entzogen sind. Die Ventilationsgrösse der Lungen endlich schwankte bei den untersuchten Personen zwischen 4,5-9,0 l, sie war annähernd dem Gewicht proportional.

Gehen wir nunmehr zu den Resultaten über, welche durch die vorliegende Untersuchung in Bezug auf den Einfluss des Weingeistes gewonnen sind, so ist es am zweckmässigsten, die bei den verschiedenen Versuchspersonen gewonnenen Ergebnisse einzeln durchzugehen.

Vorauf ist die Bemerkung zu schicken, dass vor und nach der Verabreichung grösserer Alkoholdosen die Körpertemperatur gemessen wurde. Ein Sinken iber $0,5^{\circ} \mathrm{C}$. wurde nie constatirt, und ein solches beeinflusst den Umfang der Oxydationen nicht wesentlich.

Am klarsten liegen die Verhältnisse bei Me. Die geringste Alkoholdosis war $30 \mathrm{ccm}$, die grösste $75 \mathrm{ccm}$; letztere hatte bereits deutlich berauschenden Effect. Indess es zeigte sich, dass die Sauerstoffaufnahme gänzlich ungeändert blieb, denn die geringen Schwankungen, welche das eine Mal positiv, das andere Mal negativ bei diesen Versuchen auftraten, fallen noch in das Bereich des Normalen. Hingegen zeigte sich, dass bei den grösseren Alkoholdosen die Menge der ausgeschiedenen Kohlensäure sank (von $290 \mathrm{ccm}$ auf $265 \mathrm{ccm}$, 
von 248 auf $217 \mathrm{ccm}$ ), ein Factum, auf dessen Bedeutung ich weiter unten zurtickkommen werde.

Aehnlich liegen die Verhältnisse bei $\mathrm{Kr}$. Abgesehen von der letzten Reihe, die aus im Anhang mitgetheilten Grinden nicht ganz beweisend ist, sehen wir eine in Betracht kommende $\mathrm{Zu}$ - oder $\mathrm{Ab}$ nahme des Sauerstoffconsums nicht auftreten. Auch hier wurde mit der Dosis Alkohol bis zum berauschenden Effect gestiegen. Die Werthe im Normalzustand sind bald etwas höher, bald etwas niedriger als nach Einnahme des Alkohols; schien einmal eine iber die Grenze des Normalen gehende Vermehrung vorzukommen, so bewies das Controlexperiment, dass doch nur eine Täuschung vorlag und factisch der Sauerstoffconsum sich innerhalb der normalen Grenzen als constant erwies. Dabei wurden speciell diese Versuche über eine relativ lange Zeit, bis zu 2 Stunden nach Einnahme des Alkohols, ausgedehnt.

Auch die ausgeschiedenen Kohlensäuremengen zeigten unter dem Einfluss des Alkohols keine wesentlichen Veränderungen. Ob Alkohol oder Wein resp. Cognac genommen wurde, machte keinen wesentlichen Unterschied.

Dass auch die Wirkung grosser Dosen Weingeist (125-190 cem) auf einen an starken Alkoholgenuss gewöhnten Körper keine andere ist, als die bisher betrachtete, zeigen die an Li. angestellten Experimente. Die grösste auftretende Veränderung beträgt kaum 8 bis 9 Proc. (siehe die Bemerkungen im Anhang) des gesammten Sanerstoffwechsels. Die Kohlensäureproduction zeigte einmal einen etwas bedeutenderen Abfall (circa 11 Proc.), sonst wurde auch sie nicht wesentlich beeinflusst.

Gehen wir endlich zu den beiden an Mo. angestellten Versuchsreihen über (es war zu meinem Bedauern aus äusseren Grunden nicht möglich, mehr Experimente anzustellen), so ergiebt sich aus der ersten Reihe, dass die Wirkung einer mässigen Dosis Alkohol $(30 \mathrm{ccm})$ auch auf den nicht an Alkohol gewöhnten Körper keine Wirkung hinsichtlich des Gaswechsels ausübt, anders dagegen eine berauschende Quantität. Nachdem $50 \mathrm{ccm}$ absoluter Alkohol auf einmal genommen waren, trat die berauschende Wirkung deutlich hervor, sowohl psychisch als körperlich. Unter ibrem Einflusse stieg dann, und zwar sofort, für einige Zeit (etwa 50 Minuten) die Sauerstoffaufnabme von $237 \mathrm{ccm}$ pro Minute auf $297 \mathrm{ccm}$, also um etwa 25 Proc., fiel dann allerdings schnell wieder $a b$ auf $263 \mathrm{ccm}$.

Ich war nicht in der Lage, dieses Experiment noch einmal zu wiederholen und es ist ausserordentlich schwierig, gerade für diesen 
Versuch eine geeignete Person zu finden, denn die meisten nicht an Alkoholgenuss gewöhnten Leute reagiren auf eine berauschende Quantität Alkohol mit solcher psychischer und körperlicher Unruhe, dass ein beweisender Versuch nicht mehr möglich ist. Auf der anderen Seite ist natürlich e in Experiment nicht hinreichend, einen bindenden Schluss zu gestatten.

Mit dieser einen Ausnahme aber, dass möglicherweise eine berauschende Quantität bei dem des Alkohols Ungewohnten eine ziemlich schnell vorübergehende Steigerung des Sauerstoffconsums bewirkt, können wir als wesentliches Ergebniss constatiren, dass die angewandten Dosen Alkohol einen in Betracht kommenden Effect auf die Sauerstoffaufnahmenicht hatten, dass also ein in Betracht kommendes Plus oder Minus von Oxydationen für den menschlichen Organismus nicht zu constatiren ist.

Die Kohlensäureausscheidung war entweder constant oder sie ging etwas herunter.

Fragen wir zum Schluss nach der Erklärung der gewonnenen Resultate, so ist dieselbe nach unseren heutigen physiologischen Ansehauungen eine sehr einfache. $\left.{ }^{1}\right)$ Die Menge Sauerstoff, welche in der Ruhe dem Körper zur Verfügung steht, ist eine annähernd constante Zahl. Der Sauerstoffconsum kann gesteigert werden durch Muskel- oder Driisenarbeit, nicht aber durch einfache Einführung oxydablen Materials. Die eingeführten verbrennbaren Stoffe werden aufgebraucht und dafür andere vor der Oxydation geschützt. Genau demnach, wie jeder andere Nahrungsstoff, verhält sich beim Menschen der Alkohol. Ein Theil des disponiblen Sauerstoffes, der sonst zur Oxydation anderer Stofie dienen würde, wird zur Verbrennung des Alkobols benutzt, andere Stoffe dafür gespart, wie dies Voit bereits angenommen hat ${ }^{2}$, und auf diesem Wege erklären sich auch jene öfters beobachteten Minderausscheidungen an Kohlensäure. Denn dasselbe Quantum Sauerstoff liefert je nach der chemischen Zusammensetzung des oxydirten Körpers eine sehr verschiedene Menge Kohlensäure.

1) Siehe Speck, Dieses Archiv. II. Bd. S. 412 und v. Mering und Zuntz, Archiv für die gesammte Physiologie. XV. Bd. S. 634. XXX1L. Bd. 1883.

2) Handbuch der Physiologie von L. Hermann. I. Theil. Physiologie des allgemeinen Stoffwechsels und der Ernährung von C. von Voit. S. 416. „Es fragt sich also, ob der Alkohol eine Aenderung im Stoffverbrauch hervorbringt. Nach den früheren Darlegungen ist dies allerdings der Fall: er schützt in mässigen Dosen etwas Eiweiss vor der Zersetzung und spart auch wahrscheinlich etwas Fett." 
Während $100 \mathrm{ccm}$ Sauerstoff bei der Oxydation des Alkohols nur $66 \mathrm{ccm}$ Kohlensäure liefern, ist dies Verhältniss für die meisten sonst im Körper verbrannten Stoffe (Eiweiss, Fette, Kohlehydrate) ein wesentlich anderes, d. h. auf das gleiche Quantum aufgenommenen Sauerstoffes wird bedentend mehr Kohlensäure als beim Weingeist ausgeschieden, so dass sich im Durchschnitt für den Menschen (siehe auch die Daten im Anhang) dies Verhältniss wie $80-90$ : 100 stellt.

Wieso das eine Mal diese Wirkung des Alkohols auf die Ausscheidung der Kohlensäure so prägnant, das andere Mal dagegen iberhaupt nicht hervortritt, ist zweifelhaft. Offenbar kommt hier eine Reihe einzelner Momente in Betracht, die wir nicht alle beherrschen; das Verhältniss, in welchem die im Körper kreisende Menge Alkohol zu den übrigen zur selben Zeit oxydirten Stoffen steht, die Geschwindigkeit, mit welcher er zunächst aufgenommen, dann aber verbrannt wird u. s. w., sind Factoren, die bei jedem Experiment und jedem Individuum sich verschieden gestalten können.

Es tritt hier auch am Menschen dasselbe Verhältniss hervor, welches für das Thier schon lange bekannt ist, dass nämlich die Ausscheidung der Kohlensäure von sehr viel complicirteren Bedingungen beherrscht wird, als die Aufnahme des Sauerstoffes. Von um so höherem Werth war es bei der vorliegenden Untersuchung, auch die Werthe des Sauerstoffconsums zu kennen; sie haben zum Schluss die Entscheidung gegeben, während nach den Kohlensäurewerthen wohl kaum eine so bindende Lösung der vorliegenden Frage sich hätte geben lassen.

Zum Schluss ist es mir eine angenehme Pflicht, Herrn Geh. Rath Binz für die Unterstittzung, die er mir bei meiner Arbeit hat zu Theil werden lassen, meinen herzlichsten Dank zu sagen.

\section{ANHANG.}

Versuchsdaten.

Im Nachfolgenden sind die Versuchsprotokolle und die etwa zu einzelnen Experimenten gehörigen Bemerkungen enthalten. Die Versuchsprotokolle sind in der Art wiedergegeben, dass für jede Versuchsperson eine Tabelle mitgetheilt wird, in welcher die nothwendigsten Daten wiedergegeben sind: Zusammensetzung und Menge der Exspirationsluft, Sauerstoffdeficit, Kohlensäureproduction, Sauerstoffaufnahme und endlich das Verhältniss der abgegebenen Kohlensäure zum aufgenommenen Sauerstoff (Q). 


\section{TABELLEN.}

Die Abkurzung L.V. im Folgenden bedeutet Lungenventilation, d. h. also die in einer

Minute die Lunge passirende Luftmenge.

\section{TABELle I.}

Versuchsperson Kr. 32 Jahre alt, 59 Kilo schwer, von gracilem Körperbau.

\begin{tabular}{|c|c|c|c|c|c|c|c|c|c|c|c|c|c|c|}
\hline 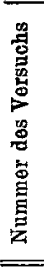 & $\begin{array}{l}\text { 泀 } \\
\text { 芴 }\end{array}$ & 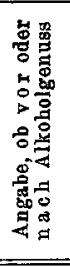 & $\begin{array}{l}\text { 葛 } \\
\stackrel{\text { S }}{2}\end{array}$ & 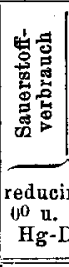 & 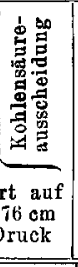 & 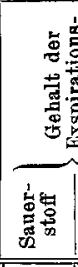 & 葛量 & 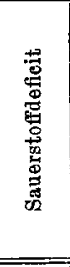 & 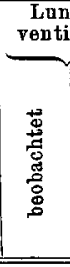 & 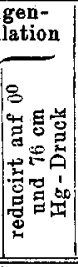 & $\%$ & 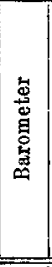 & 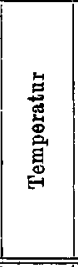 & 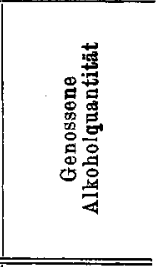 \\
\hline & & & & & & & & & & & & & Grad & \\
\hline a & $7 /$ V. 86 & vor & $12^{17}-12^{53}$ & 203,5 & 178,9 & 17,21 & 3,36 & 3,82 & 5,68 & 5,33 & 0,88 & 763 & & \\
\hline b & & vor & $4^{52}-5^{36}$ & 192,8 & 158,2 & 17,39 & 3,02 & 3,68 & 5,62 & 5,24 & 0,82 & 763 & 15,2 & \\
\hline $\mathrm{c}$ & & nach & $6^{01}-6^{23}$ & 236,6 & 189,6 & 16,98 & 3,30 & 4,12 & 6,16 & 5,75 & 0,80 & $\Rightarrow$ & $=$ & $30 \mathrm{ccm}$ \\
\hline $\mathrm{d}$ & & nach & $6^{28}-7^{15}$ & 219,5 & 175,1 & 17,09 & 3,20 & 4,01 & 5,87 & 5,45 & 0,80 & $=$ & $=$ & \\
\hline e & $12 / \mathrm{V}$ & vor & $12^{06}-1^{32}$ & 226,3 & 159,6 & 15,56 & 3,14 & 4,45 & 5,57 & 5,08 & 0,71 & 749 & 16,0 & \\
\hline$f$ & & vor & $4^{29}-5^{01}$ & 222,9 & 184,6 & 17,08 & 3,30 & 3,99 & 6,13 & 5,60 & 0,82 & $=$ & 16,2 & \\
\hline $\mathrm{g}$ & & vor & $5^{06}-5^{27}$ & 227,2 & 192,7 & 16,98 & 3,46 & 4,08 & 6,10 & 5,57 & 0,84 & $=$ & $=$ & \\
\hline h & & nach & $5^{45}-6^{30}$ & 219,0 & 182,0 & 16,93 & 3,45 & 4,14 & 5,80 & 5,29 & 0,83 & $=$ & $=$ & 50 \\
\hline i & $20 / V$ & nach & $6^{33}-7^{13}$ & 220,4 & 166,4 & 16,79 & 3,29 & 4,36 & 5,54 & 5,04 & 0,75 & $=$ & $=$ & \\
\hline $\mathbf{k}$ & & vor & $4^{20}-5^{04}$ & 214,1 & $\mid 163,9$ & 16,88 & 3,26 & 4,26 & 5,50 & 5,03 & 0,76 & 759 & 18,2 & \\
\hline 1 & & nach & $5^{21}-6^{04}$ & 221,0 & 161,2 & 17,13 & 2,94 & 4,03 & 6,00 & 5,50 & 0,72 & $=$ & $=$ & 50 \\
\hline $\mathrm{m}$ & $5 / \mathrm{VI}$. & nach & $6^{09}-6^{36}$ & 207,2 & 168,0 & 17,02 & 3,30 & 4,07 & 5,57 & 5,09 & 0,81 & $=$ & $=$ & \\
\hline $\mathbf{n}$ & & vor & $\begin{array}{l}5^{51}-6^{44} \\
058\end{array}$ & 196,1 & 164,0 & 17,06 & 3,35 & 4,01 & 5,40 & 4,90 & 0,84 & 755 & 18,9 & \\
\hline o & & nach & $60-70$ & 221,3 & $\mid 120, \mathrm{~b}$ & 16,80 & 3,27 & 4,36 & 5,75 & 5,21 & 0,75 & $i=$ & $=$ & 70 \\
\hline $\mathbf{p}$ & 9/VI. & $\begin{array}{c}\text { naen } \\
\text { yor }\end{array}$ & $101-8$ & 197,0 & 154,9 & 10,90 & 3,26 & 4,16 & 5,24 & 4,75 & 0,78 & $=0$ & $\Rightarrow$ & \\
\hline$q$ & & vor & $1^{21}-1^{00}$ & 209,7 & 177,2 & 17,00 & 3,43 & 4,06 & 5,74 & 5,17 & 0,85 & 752 & 19,5 & \\
\hline $\mathbf{r}$ & & nach & $5^{14}-6^{03}$ & 187,6 & 168,3 & 17,10 & 3,51 & 3,91 & 5,33 & 4,80 & 0,90 & $=$ & $=$ & \\
\hline $\mathbf{s}$ & & $\begin{array}{l}\text { nach } \\
\text { nach }\end{array}$ & $6^{16}-7^{01}$ & 205,1 & 176,3 & & & 3,78 & 6,03 & 5,43 & 0,86 & $x$ & $=$ & 70 \\
\hline$t$ & & $\begin{array}{l}\text { nach } \\
\text { nach }\end{array}$ & $7^{05}-7^{52}$ & 198,7 & 170,6 & 17,22 & 3,28 & 3,82 & 5,78 & 5,20 & $\mid 0,86$ & $=$ & $=$ & \\
\hline $\mathrm{u}$ & $12 / \mathrm{VI}$. & nach & $7^{55}-8^{48}$ & 208,0 & 169,4 & 16,94 & 3,38 & 4,15 & 5,57 & 5,01 & 0,81 & $=$ & $=$ & \\
\hline $\mathbf{v}$ & & nach & $12^{31}-1^{27}$ & 178,6 & 145,5 & 16,67 & 3,61 & 4,43 & 4,47 & 4,03 & 0,81 & 755 & 19,8 & \\
\hline W & & nach & $1^{33}-2^{27}$ & 207,6 & 161,7 & 16,48 & 3,63 & 4,66 & 4,94 & 4,46 & 0,78 & $=$ & $=$ & $25=$ \\
\hline $\bar{x}$ & & vor & $5^{42}-6^{36}$ & 227,6 & 175,4 & 16,41 & 3,66 & 4,75 & 5,26 & 4,79 & 0,77 & 758 & 18,6 & (inPortwein) \\
\hline$\Delta$ & & nach & $6^{51}-7^{38}$ & 215,3 & 171,9 & 16,89 & 3,37 & 4,22 & 5,60 & 5,10 & 0,79 & $=$ & $=$ & $60 \mathrm{ccm}$ \\
\hline $\mathrm{y}$ & & nach & $7^{42}-8^{36}$ & 215,0 & 163,1 & 16,56 & 3,49 & 4,60 & 5,13 & 4,67 & 0,76 & $=$ & $=$ & (in Cognac) \\
\hline $\mathbf{z}$ & $17 / V I$. & vor & $5^{02}-5^{53}$ & 213,6 & 178,3 & 16,72 & 3,64 & 4,36 & 5,38 & 4,90 & 0,83 & 754 & 17,06 & \\
\hline aa & & nach & $6^{15}-7$ & - & - & - & - & - & 5,90 & - & - & $=$ & $=$ & $75 \mathrm{ccm}$ \\
\hline $\mathrm{bb}$ & & nach & $7^{11}-8$ & 217,5 & 164,3 & 16,69 & 3,39 & 4,49 & 5,32 & 4,85 & 6,76 & $=$ & $=$ & (in Cognac) \\
\hline $\mathrm{ec}$ & 9/VII. & vor & $4^{07}-5^{03}$ & 203,0 & 169,2 & 16,65 & 3,70 & 4,44 & $\mathbf{5 , 0 7}$ & 4,57 & 0,83 & 756 & 19,8 & \\
\hline dd & & nach & $5^{16}-6^{04}$ & 243,0 & 192,2 & 16,58 & 3,60 & 4,55 & 5,92 & 5,34 & 0,79 & $=$ & $=$ & $60 \mathrm{ccm}$ \\
\hline $\begin{array}{l}\mathrm{ee} \\
\text { ff }\end{array}$ & & nach & $6^{07}-6^{56}$ & 242,2 & 187,7 & 16,44 & 3,65 & 4,71 & 5,70 & 5,14 & 0,77 & $=$ & $*$ & $\begin{array}{c}\text { (in Rheinwoin- } \\
\text { mousseux) }\end{array}$ \\
\hline
\end{tabular}

Der mittlere Sauerstoffverbrauch in der Ruhe betrug $208 \mathrm{ccm}$. Der Sauerstoffverbrauch schwankte zwischen 178,6 und $227,6 \mathrm{ccm}$. Der mittlere Sauerstoffverbrauch pro Kilo und Minute betrug $3,53 \mathrm{ccm}$. 
Die mittlere Kohlensäureausscheidung betrug $170,4 \mathrm{ccm}$. Die Kohlensäureausscheidung schwankt zwischen 145,5 and $192,7 \mathrm{~cm}$. Die mittlere Kohlensäureausscheidung pro Kilo und Minute betrug $2,88 \mathrm{ccm}$.

TABellae II.

Versuchsperson Me. 24 Jahre alt, 96 Kilo schwer, ziemlich fettreiches Individuum.

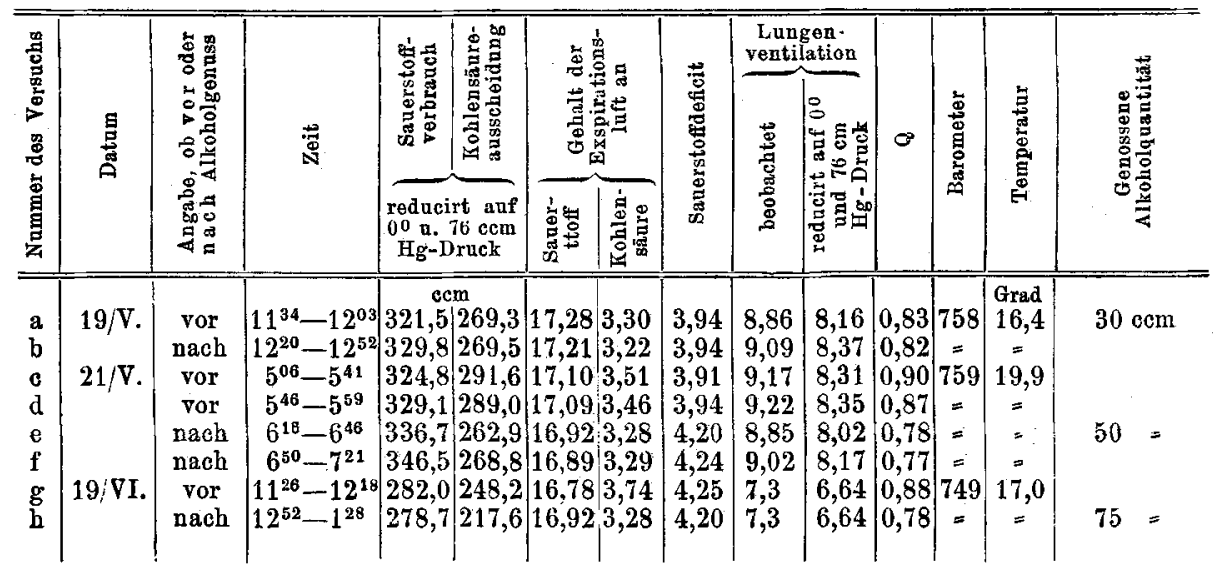

Die mittlere Sauerstoffaufnahme beträgt $314,5 \mathrm{ccm}$, sie schwankt zwischen 282,0 und $329,1 \mathrm{ccm}$. Die mittlere Kohlensäureausscheidung betrug $219,6 \mathrm{ccm}$, sie schwankte zwischen 248,2 und $289,0 \mathrm{ccm}$. Der mittlere Sauerstoffverbrauch pro Kilo und Minute betrug 3,27 ccm. Die mittlere Kohlensäureausscheidung betrug $2,28 \mathrm{~cm}$ pro Kilo und Minute.

TABELLE III.

Versuchsperson Mo. Ca. 30 Jahre alt. Nicht an Alkoholgenuss gewöhnt.

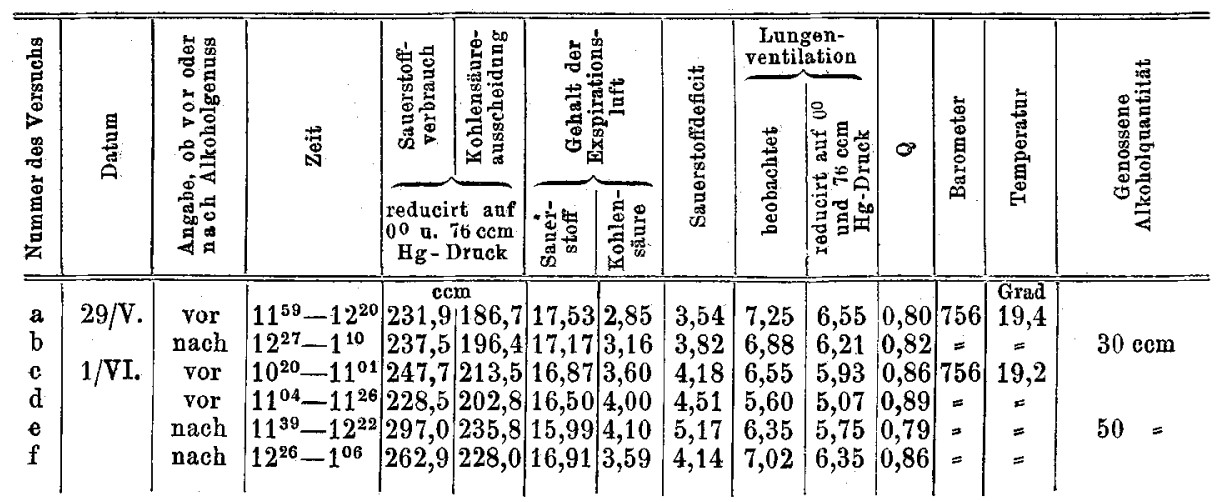

Die mittlere Sauerstoffaufnahme betrug $236 \mathrm{ccm}$, die mittlere Kohlensäureausscheidung $208 \mathrm{ccm}$. 


\section{TABelle IV.}

Versuchsperson Li. 25 Jahre alt, 72 Kilo schwer, fettarm und musculös. Arbeiter, Potator.

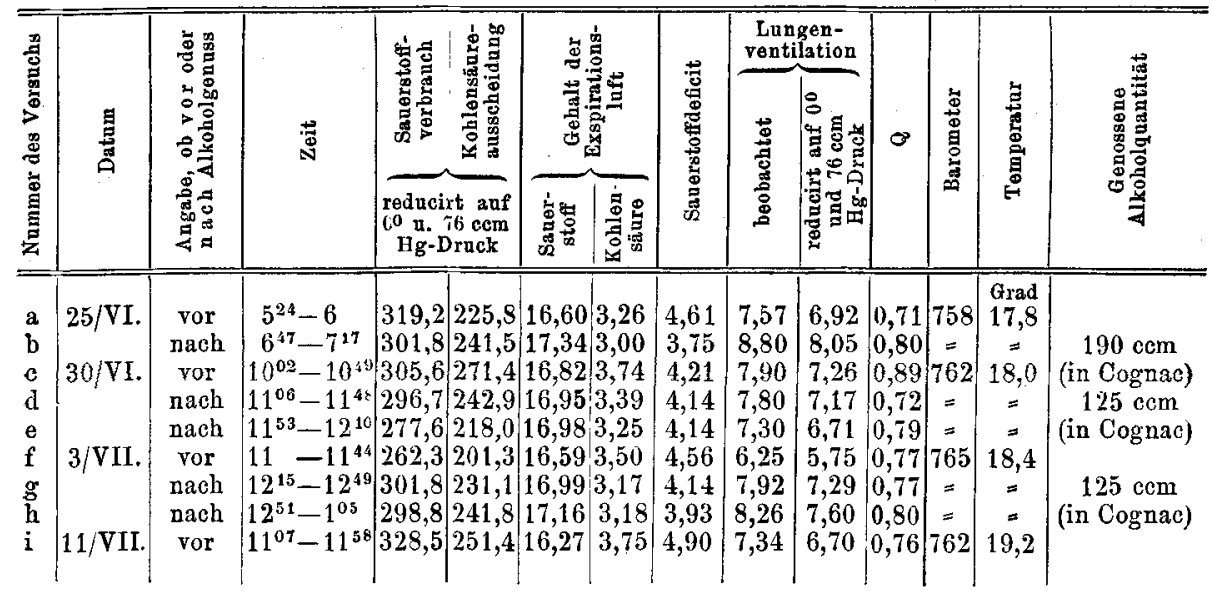

Die mittlere Sauerstoffaufnahme betrug $317,7 \mathrm{ccm}$, schwankte zwischen 305,6 und $328,5 \mathrm{ccm}$; die mittlere Kohlensäureausscheidung 249,5, schwankte $\mathrm{zwischen} 325,8$ und $271,4 \mathrm{ccm}$. Der mittlere Sauerstoffverbrauch pro Kilo und Minute betrug 4,41 ccm. Die mittlere Kohlensäureausscheidung betrug $3,47 \mathrm{ccm}$.

\section{Zu Tabelle I.}

Reihe ee und ff. Es waren $350 \mathrm{ccm}$ Rheinweinmousseux $=52 \mathrm{ccm}$ Alcoh. abs. genommen. Beim Liegen auf dem Sopha trat infolge der fortwährenden Neigung zum Aufstossen Unbehagen auf, daher war die Lage nicht ganz ruhig. In diesem Umstand ist wohl der Grund fur die Erhöhung des Sauerstoffconsums zu suchen.

\section{Zu Tabelle II.}

Reihe f. Während dieser Reihe lag Me. 6 Minuten lang nicht ganz ruhig. Die Athemgrösse, welche sonst zwischen 8,5-8,9 1 schwankte, stieg während dieser Zeit auf 9,4 im Durchschnitt. Daher rührt wohl die allerdings auch an sich geringe Erhöhung des Gaswechsels.

\section{$\mathrm{Zu}$ Tabelle IV.}

Reihe e. Li. schläft ziemlich fest ein, woher sich die geringen Werthe des Gaswechsels erklären.

Reihe f. Die Athemgrössen pro Minute waren folgende: $8,1-8,0$ $-7,2-6,5-6,3-5,6-5,4-5,5-4,8-5,0-4,8-4,6-8,3-7,6-8,5$ $-8,0-8,4-8,8$ u. s. w. 
384 XVII. GEPPERT, Einwirkung des Alkohols auf den Gaswechsel des Menschen.

Nach den ersten, 3 Werthen (entsprechend 6 Minuten) trat Schlaf ein; bei dem Werth 8,5 hörte er wieder auf. Daher rühren die niedrigen Werthe für den Sauerstoffconsum. Da am selben Tage aus äusseren Gründen der Versuch nicht allzu lange fortgesetzt werden durfte, so wurde später (Reihe i) noch eine Normalbestimmung im wachen Zustand ausgeführt.

Bonn, October 1886.

\section{NACHTRAG.}

Nachdem vorstehende Arbeit bereits zur Publication eingesandt war, erschien eine Mittheilung von $N$. Zuntz und Berdez ${ }^{1}$ ) über dasselbe Thema. $\mathrm{Zuntz}$ und Berdez constatirten in 4 Versuchen, dass die Sauerstoffaufnahme des Menschen nach Einnahme von 20 bis $30 \mathrm{ccm}$ Ale. absol. eine sehr geringe Zunahme (3,5 Proc. im Mittel) erfuhr. Aehnliche Zunahmen (siehe die Daten der Tabellen) habe auch ich manchmal beobachtet; da sie indess inconstant waren und auch Verminderungen vorkamen, ist es mir fraglich, ob auf so geringe Zunahmen Gewicht zu legen ist. Hinsichtlich der theoretischen Deutung der Versuche stimme ich mit Zuntz und Berdez vollkommen uberein.

1) Fortschritte der Medicin. 1887. Heft 1: Beitrag zur Kenntniss der Einwirkung des Weingeistes auf den Respirationsprocess des Menschen. Nach Versuchen mit Dr. Berdez aus Lausanne referirt von Prof. N. Zuntz in Berlin. 